ESAIM: PROCEEDINGS AND SURVEYS, September 2014, Vol. 45, p. 502-511

J.-S. Dhersin, Editor

\title{
FINITE-VOLUME METHOD FOR THE CAHN-HILLIARD EQUATION WITH DYNAMIC BOUNDARY CONDITIONS
}

\author{
FLORE NABET ${ }^{1}$
}

\begin{abstract}
A numerical scheme is proposed here to solve a diphasic Cahn-Hilliard equation with dynamic boundary conditions. A finite-volume method is implemented for the space discretization and existence and convergence results are proved. Numerical simulations are also presented that show the influence of these boundary conditions.
\end{abstract}

\section{INTRODUCTION}

The Cahn-Hilliard equation describes the evolution of binary mixtures when, for example, a binary alloy is cooled down sufficiently. This problem has been extensively studied for many years with Neumann boundary conditions. Recently, physicists [5-7] have introduced new boundary conditions, usually called dynamic boundary conditions, to account for the effective interaction between the wall and the two mixture components in a confined system. With these dynamic boundary conditions, the Cahn-Hilliard equation results in the following system: Find the concentration $c:[0, T[\times \Omega \rightarrow \mathbb{R}$ such that:

$$
\left\{\begin{aligned}
\partial_{t} c & =\Gamma_{b} \Delta \mu, & & \text { in }(0, T) \times \Omega ; \\
\mu & =-\varepsilon \sigma_{b} \Delta c+\frac{\sigma_{b}}{\varepsilon} f_{b}^{\prime}(c), & & \text { in }(0, T) \times \Omega ; \\
c(0, .) & =c_{0}, & & \text { in } \Omega ; \\
\frac{\varepsilon^{3}}{\Gamma_{s} \Gamma_{b}} \partial_{t} c_{\Gamma} & =\varepsilon^{2} \sigma_{s} \sigma_{b} \Delta_{\|} c_{\Gamma}-\sigma_{b} f_{s}^{\prime}\left(c_{\Gamma}\right)-\varepsilon \sigma_{b} \partial_{n} c, & & \text { on }(0, T) \times \Gamma ; \\
\partial_{n} \mu & =0, & & \text { on }(0, T) \times \Gamma ;
\end{aligned}\right.
$$

where $\mu$ is an intermediate unknown called chemical potential. There cannot be any mass exchange through the boundary, which is why we consider the homogeneous Neumann boundary condition for the chemical potential. The domain $\Omega \subset \mathbb{R}^{2}$ is smooth, connected and bounded, with $\Gamma=\partial \Omega$ its boundary and $T>0$ the final time. The Laplace-Beltrami operator on $\Gamma$ is noted $\Delta_{\|}, \partial_{n}$ is the normal derivative at the boundary and $c_{\Gamma}$ is the trace of $c$ on $\Gamma$.

These dynamic boundary conditions induced us to look for a solution in $L^{\infty}\left(0, T, H^{1}(\Omega)\right)$ whose trace is in $L^{\infty}\left(0, T, H^{1}(\Gamma)\right)$ (see Theorem 4.5).

The parameter $\varepsilon>0$ accounts for the interface thickness, the coefficient $\Gamma_{b}>0$ is the bulk mobility and $\sigma_{b}>0$ is the fluid-fluid surface tension. On the boundary, $\Gamma_{s}>0$ defines a surface kinetic coefficient and $\sigma_{s}>0$ a surface capillarity coefficient. The nonlinear terms $f_{b}$ and $f_{s}$ represent respectively the bulk free energy density and the surface free energy density and they satisfy the following assumptions:

1 Aix-Marseille Université, CNRS, Centrale Marseille, LATP, UMR 7353, 13453, Marseille, France (flore.nabet@univ-amu.fr).

(C) EDP Sciences, SMAI 2014 
- Dissipativity:

$$
\liminf _{|x| \rightarrow \infty} f_{b}^{\prime \prime}(x)>0 \quad \text { and } \quad \liminf _{|x| \rightarrow \infty} f_{s}^{\prime \prime}(x)>0 .
$$

- Polynomial growth for $f_{b}$ : there exist $C_{b}>0$ and a real $p \geq 2$ such that:

$$
\begin{aligned}
\left|f_{b}(x)\right| & \leq C_{b}\left(1+|x|^{p}\right), \\
\left|f_{b}^{\prime}(x)\right| & \leq C_{b}\left(1+|x|^{p-1}\right), \\
\left|f_{b}^{\prime \prime}(x)\right| & \leq C_{b}\left(1+|x|^{p-2}\right) .
\end{aligned}
$$

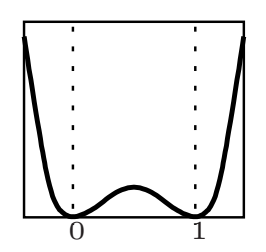

Figure 1. Typical choice for $f_{b}: f_{b}(c)=c^{2}(1-c)^{2}$.

Remark 1.1. We can notice that if we choose $\sigma_{s}=0, \Gamma_{s}=+\infty$ and $f_{s}=0$, we formally recover the standard Neumann boundary condition $\partial_{n} c=0$.

The Cahn-Hilliard equation with dynamic boundary conditions $(\mathcal{P})$ is such that the total free energy functional defined by

$$
\mathcal{F}(c)=\int_{\Omega}\left(\frac{\varepsilon}{2} \sigma_{b}|\nabla c|^{2}+\frac{\sigma_{b}}{\varepsilon} f_{b}(c)\right)+\int_{\Gamma}\left(\frac{\varepsilon^{2}}{2} \sigma_{s} \sigma_{b}\left|\nabla_{\|} c_{\Gamma}\right|^{2}+\sigma_{b} f_{s}\left(c_{\Gamma}\right)\right),
$$

is decreasing with respect to time:

$$
\frac{d}{\mathrm{~d} t} \mathcal{F}(c(t, .))=-\Gamma_{b} \int_{\Omega}|\nabla \mu(t, .)|^{2}-\frac{\varepsilon^{3}}{\Gamma_{s} \Gamma_{b}} \int_{\Gamma}\left|\partial_{t} c_{\Gamma}(t, .)\right|^{2}, \quad t \in[0, T[.
$$

From a mathematical point of view, problem $(\mathcal{P})$ has already been studied in [9-11] where questions such as global existence and uniqueness, existence of a global attractor, maximal regularity of solutions and convergence to an equilibrium have been answered. From a numerical point of view, some numerical schemes have been considered in [5-7] in a finite-difference framework but without proof of convergence. In [2], the authors propose a spatial finite-element semi-discretization and prove error estimate and convergence results on a slab with periodic conditions in the lateral directions and dynamic conditions in the vertical directions, so that complex geometries of the domain are not taken into account in the convergence analysis.

In this paper, we investigate a finite-volume scheme for the space discretization of this problem. This method is well adapted to the coupling of the dynamics in the domain and those on the boundary by the flux term $\partial_{n} c$. Moreover, this kind of scheme preserves the mass and accounts naturally for the non-flat geometry of the boundary and for the associated Laplace-Beltrami operator. In Section 2, we recall the main finite-volume notation, for example used in [4], that we adapt to our problem with a curved domain and dynamic boundary conditions. In Section 3, we give the discrete energy functional and the associated energy estimates. Then, we propose a finite-volume scheme with different time discretizations for the nonlinear terms. Existence and convergence results are stated in Section 4. Finally, we give some numerical results in Section 5 with different nonlinear terms on the boundary.

\section{THE DISCRETE FRAMEWORK}

We give in this section the main notation and definitions used in this paper. 


\subsection{The discretization}

Since $\Omega$ is a curved domain, the notation (Fig. 2) and definitions are slightly different from the usual finitevolume definitions given for example in [4].

An admissible mesh $\mathcal{T}$ is constituted of an interior mesh $\mathfrak{M}$ and a boundary mesh $\partial \mathfrak{M}$. The interior mesh $\mathfrak{M}$ is a set of control volumes (we specify that some control volumes are curved) $\mathcal{K} \subset \Omega$ such that:

- if $\mathcal{K} \neq \mathcal{L}$, we have $\mathcal{K} \cap \stackrel{\mathcal{L}}{=} \emptyset$;

- if $\mathcal{K} \neq \mathcal{L}$ such that the dimension of $\overline{\mathcal{K}} \cap \overline{\mathcal{L}}$ is equal to 1 , then $\overline{\mathcal{K}} \cap \overline{\mathcal{L}}$ is an edge of the mesh;

- $\cup_{\mathcal{K} \in \mathfrak{M}} \overline{\mathcal{K}}=\bar{\Omega}$.

We note $\partial \mathfrak{M}$ the set of edges of the control volumes in $\mathfrak{M}$ included in $\Gamma$ (we remark that these are not segments but curved sections). We will use two different notations for an element of $\partial \mathfrak{M}$ : we note e when we consider it as a control volume belonging to $\partial \mathfrak{M}$ and we note $\sigma$ when we consider it as the edge of an interior control volume $\mathcal{K} \in \mathfrak{M}$.

Let $\mathcal{E}$ be the set of the edges of the mesh $\mathcal{T}, \mathcal{E}_{\text {ext }}=\partial \mathfrak{M}$ is the set of exterior edges and $\mathcal{E}_{\text {int }}=\mathcal{E} \backslash \mathcal{E}_{\text {ext }}$ is the set of interior edges. Let $m_{\sigma}$ be the length of the edge $\sigma \in \mathcal{E}$.

For each control volume $\mathcal{K} \in \mathfrak{M}$, we associate a point $x_{\mathcal{K}} \in \mathcal{K}$ and we assume that for all neighbouring control volumes $\mathcal{K}, \mathcal{L} \in \mathfrak{M}$ the edge $\sigma=\mathcal{K} \mid \mathcal{L} \in \mathcal{E}_{\text {int }}$ is orthogonal to the straight line going through $x_{\mathcal{K}}$ and $x_{\mathcal{L}}$. The distance between $x_{\mathcal{K}}$ and $x_{\mathcal{L}}$ is noted $d_{\mathcal{K}, \mathcal{L}}$ and $\overrightarrow{\mathbf{n}}_{\mathcal{K} \mathcal{L}}$ is the unit normal vector going from $\mathcal{K}$ to $\mathcal{L}$. We define by $\widetilde{\mathcal{K}}$ the polygon shaped by the vertices of $\mathcal{K}$ if there exists at least an edge of $\mathcal{K}$ on the boundary and, if not, $\widetilde{\mathcal{K}}=\mathcal{K}$. Let $m_{\mathcal{K}}$ (respectively $m_{\widetilde{\mathcal{K}}}$ ) be the Lebesgue measure of $\mathcal{K}$ (respectively $\widetilde{\mathcal{K}}$ ).

For any $\mathbf{e} \in \partial \mathfrak{M}$, we note $\widetilde{\mathbf{e}}$ the chord associated with $\mathbf{e}, m_{\widetilde{\mathbf{e}}}$ its length. We define $x_{\mathbf{e}}$ as the intersection between $\Gamma$ and the straight line passing throught $x_{\mathcal{K}}$ and orthogonal to $\widetilde{\mathbf{e}}$. Let $y_{\mathbf{e}}$ be the intersection between the line $\left(x_{\mathcal{K}} x_{\mathbf{e}}\right)$ and the chord $\widetilde{\mathbf{e}}$. We note $d_{\mathcal{K}, \mathbf{e}}$ the distance between $x_{\mathcal{K}}$ and $y_{\mathbf{e}}$ and $\overrightarrow{\mathbf{n}}_{\mathcal{K} \mathbf{e}}$ is the unit normal vector to $\widetilde{\mathbf{e}}$ outward to $\mathcal{K}$.

Let $\mathcal{V}$ be the set of the vertices included in $\Gamma$ and $d_{\mathbf{e}, \mathbf{v}}$ be the distance between the center $y_{\mathbf{e}}$ and the vertex $\mathbf{v} \in \mathcal{V}$. For a vertex $\mathbf{v}=\mathbf{e} \mid \mathbf{e}^{\prime} \subset \Gamma$ which separates the control volumes $\mathbf{e}, \mathbf{e}^{\prime} \in \partial \mathfrak{M}, d_{\mathbf{e}, \mathbf{e}^{\prime}}$ is equal to the sum of $d_{\mathbf{e}, \mathbf{v}}$ and $d_{\mathbf{e}^{\prime}, \mathbf{v}}$.

We can notice that the proposed scheme uses only the coordinates of the vertices of the mesh in $\Gamma$ and not the equation of the boundary $\Gamma$.

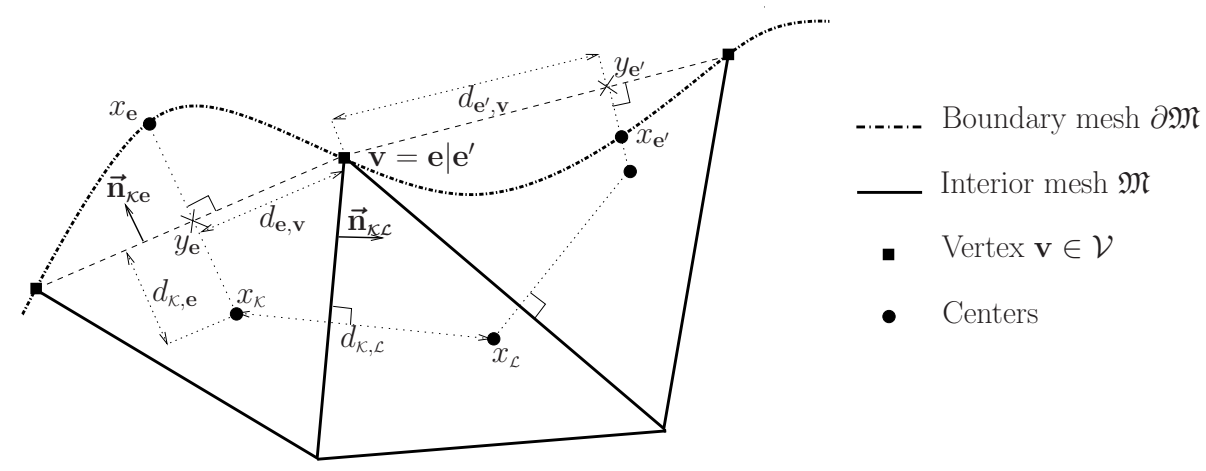

Figure 2. Finite-volume meshes

The mesh size is defined by: $\operatorname{size}(\mathcal{T})=\sup \{\operatorname{diam}(\mathcal{K}), \mathcal{K} \in \mathfrak{M}\}$. All the constants in the results below depend on a certain measure of the regularity of the mesh, which is classical and that we do not make explicit here in order to be more synthetic. In short, it is necessary that the control volumes do not become flat when the mesh is refined.

Let $N \in \mathbb{N}^{*}$ and $\left.T \in\right] 0,+\infty[$. The temporal interval $[0, T]$ is uniformly discretized with a fixed time step $\Delta t=\frac{T}{N}$. For $n \in\{0, \cdots, N\}$, we define $t^{n}=n \Delta t$. 


\subsection{Discrete unknowns}

For a given time step $t^{n}$, the FV method associates with all interior control volumes $\mathcal{K} \in \mathfrak{M}$ an unknown value $c_{\mathcal{K}}^{n}$ and with all boundary control volumes $\mathbf{e} \in \partial \mathfrak{M}$ an unknown value $c_{\mathbf{e}}^{n}$ for the order parameter. The same notations are used for the chemical potential with an unknown value $\mu_{\mathcal{K}}^{n}$ for all $\mathcal{K} \in \mathfrak{M}$. Because of the Neumann boundary condition, it is not necessary to have boundary unknows for $\mu$.

Whenever it is convenient, we associate with a discrete function $u_{\mathcal{T}} \in \mathbb{R}^{\mathcal{T}}$ the piecewise constant functions $u_{\mathcal{T}}=\left(u_{\mathfrak{M}}, u_{\partial \mathfrak{M}}\right)$ where $u_{\mathfrak{M}}=\sum_{\mathcal{K} \in \mathfrak{M}} u_{\mathcal{K}} 1_{\mathcal{K}} \in L^{\infty}(\Omega)$ and $u_{\partial \mathfrak{M}}=\sum_{\mathbf{e} \in \partial \mathfrak{M}} u_{\mathbf{e}} 1_{\mathbf{e}} \in L^{\infty}(\Gamma)$.

We note $u_{\mathfrak{m}}^{\Delta t}$ (respectively $u_{\partial \mathfrak{m}}^{\Delta t}$ ) the piecewise constant function in $] 0, T[\times \Omega$ (respectively $] 0, T[\times \Gamma$ ) such that for all $t \in\left[t^{n}, t^{n+1}[\right.$ :

$$
u_{\mathfrak{m}}^{\Delta t}(t, x)=u_{\mathcal{K}}^{n+1} \text { if } x \in \mathcal{K} \quad \text { and } \quad u_{\partial \mathfrak{M}}^{\Delta t}(t, x)=u_{\mathbf{e}}^{n+1} \text { if } x \in \mathbf{e} .
$$

\subsection{Discrete inner products and norms}

Definition 2.1 (Discrete $L^{2}$ norms).

- For $u_{\mathfrak{M}} \in \mathbb{R}^{\mathfrak{M}}$, the $L^{2}(\Omega)$ discrete norm of $u_{\mathfrak{m}}$ is defined by:

$$
\left\|u_{\mathfrak{M}}\right\|_{0, \mathfrak{M}}^{2}=\sum_{\mathcal{K} \in \mathfrak{M}} m_{\widetilde{\mathcal{K}}} u_{\mathcal{K}}^{2} .
$$

- For $u_{\partial \mathfrak{M}} \in \mathbb{R}^{\partial \mathfrak{M}}$, the $L^{2}(\Gamma)$ discrete norm of $u_{\partial \mathfrak{M}}$ is defined by:

$$
\left\|u_{\partial \mathfrak{M}}\right\|_{0, \partial \mathfrak{M}}^{2}=\sum_{\mathbf{e} \in \partial \mathfrak{M}} m_{\widetilde{\mathbf{e}}} u_{\mathbf{e}}^{2}
$$

Definition 2.2 (Discrete $H^{1}$ semi-definite inner products).

- For $u_{\mathcal{T}}, v_{\mathcal{T}} \in \mathbb{R}^{\mathcal{T}}$, the $H^{1}(\Omega)$ discrete semi-definite inner product is defined by:

$$
\llbracket u_{\mathcal{T}}, v_{\mathcal{T}} \rrbracket_{1, \mathcal{T}}=\sum_{\sigma \in \mathcal{E}_{i n t}} m_{\sigma} d_{\mathcal{K}, \mathcal{L}}\left(\frac{u_{\mathcal{K}}-u_{\mathcal{L}}}{d_{\mathcal{K}, \mathcal{L}}}\right)\left(\frac{v_{\mathcal{K}}-v_{\mathcal{L}}}{d_{\mathcal{K}, \mathcal{L}}}\right)+\sum_{\sigma \in \mathcal{E}_{\text {ext }}} m_{\widetilde{\mathbf{e}}} d_{\mathcal{K}, \mathbf{e}}\left(\frac{u_{\mathcal{K}}-u_{\mathbf{e}}}{d_{\mathcal{K}, \mathbf{e}}}\right)\left(\frac{v_{\mathcal{K}}-v_{\mathbf{e}}}{d_{\mathcal{K}, \mathbf{e}}}\right),
$$

where, by convention, $u_{\mathbf{e}}=u_{\mathcal{K}}$ for $\sigma=\mathbf{e} \in \mathcal{E}_{\text {ext }}$ an edge of $\mathcal{K}$ if $u_{\mathcal{T}}$ satisfies the homogeneous Neumann boundary condition. We note $\left|u_{\mathcal{T}}\right|_{1, \mathcal{T}}=\llbracket u_{\mathcal{T}}, u_{\mathcal{T}} \rrbracket_{1, \mathcal{T}}^{\frac{1}{2}}$ the associated discrete $H^{1}(\Omega)$ seminorm.

- For $u_{\partial \mathfrak{M}}, v_{\partial \mathfrak{M}} \in \mathbb{R}^{\partial \mathfrak{M}}$, the $H^{1}(\Gamma)$ discrete semi-definite inner product is defined by:

$$
\llbracket u_{\partial \mathfrak{M}}, v_{\partial \mathfrak{M}} \rrbracket_{1, \partial \mathfrak{M}}=\sum_{\mathbf{v}=\mathbf{e} \mid \mathbf{e}^{\prime} \in \mathcal{V}} d_{\mathbf{e}, \mathbf{e}^{\prime}}\left(\frac{u_{\mathbf{e}}-u_{\mathbf{e}^{\prime}}}{d_{\mathbf{e}, \mathbf{e}^{\prime}}}\right)\left(\frac{v_{\mathbf{e}}-v_{\mathbf{e}^{\prime}}}{d_{\mathbf{e}, \mathbf{e}^{\prime}}}\right) .
$$

We note $\left|u_{\partial \mathfrak{M}}\right|_{1, \partial \mathfrak{M}}=\llbracket u_{\partial \mathfrak{M}}, u_{\partial \mathfrak{M}} \rrbracket_{1, \partial \mathfrak{M}}^{\frac{1}{2}}$ the associated discrete $H^{1}(\Gamma)$ seminorm.

Now, we can define the $H^{1}$ discrete norms by:

$$
\left\|u_{\mathcal{T}}\right\|_{1, \mathcal{T}}^{2}=\left\|u_{\mathfrak{M}}\right\|_{0, \mathfrak{M}}^{2}+\left|u_{\mathcal{T}}\right|_{1, \mathcal{T}}^{2}, \quad \forall u_{\mathcal{T}} \in \mathbb{R}^{\mathcal{T}} \quad \text { and } \quad\left\|u_{\partial \mathfrak{M}}\right\|_{1, \partial \mathfrak{M}}^{2}=\left\|u_{\partial \mathfrak{M}}\right\|_{0, \partial \mathfrak{M}}^{2}+\left|u_{\partial \mathfrak{M}}\right|_{1, \partial \mathfrak{M}}^{2}, \quad \forall u_{\partial \mathfrak{M}} \in \mathbb{R}^{\partial \mathfrak{M}} .
$$

\section{NumERICAL SCHEME AND ENERGY ESTIMATES}

\subsection{Numerical scheme}

We use a consistent two-point flux approximation for Laplace operators in $\Omega$ and a consistent two-point flux approximation for the Laplace-Beltrami operator on $\Gamma$. For nonlinear terms, we use two different discretizations described below, fully implicit and semi-implicit, so that we have to use a Newton method at each iteration.

We assume that $c_{\mathcal{T}}^{n} \in \mathbb{R}^{\mathcal{T}}$ is given. The scheme then writes as follows. 
Problem 3.1. Find $\left(c_{\mathcal{T}}^{n+1}, \mu_{\mathfrak{M}}^{n+1}\right) \in \mathbb{R}^{\mathcal{T}} \times \mathbb{R}^{\mathfrak{M}}$ such that $\forall u_{\mathcal{T}} \in \mathbb{R}^{\mathcal{T}}, \forall v_{\mathfrak{M}} \in \mathbb{R}^{\mathfrak{M}}$ :

$$
\left\{\begin{aligned}
\sum_{\mathcal{K} \in \mathfrak{M}} m_{\widetilde{\mathcal{K}}} \frac{c_{\mathcal{K}}^{n+1}-c_{\mathcal{K}}^{n}}{\Delta t} v_{\mathcal{K}}= & -\Gamma_{b} \llbracket \mu_{\mathfrak{M}}^{n+1}, v_{\mathfrak{M}} \rrbracket_{1, \mathcal{T}} \\
\sum_{\mathcal{K} \in \mathfrak{M}} m_{\widetilde{\mathcal{K}}} \mu_{\mathcal{K}}^{n+1} u_{\mathcal{K}}= & \varepsilon \sigma_{b} \sum_{\sigma \in \mathcal{E}_{i n t}} \frac{m_{\sigma}}{d_{\mathcal{K}, \mathcal{L}}}\left(c_{\mathcal{K}}^{n+1}-c_{\mathcal{L}}^{n+1}\right)\left(u_{\mathcal{K}}-u_{\mathcal{L}}\right) \\
& +\varepsilon \sigma_{b} \sum_{\sigma \in \mathcal{E}_{\text {ext }}} \frac{m_{\widetilde{\mathbf{e}}}}{d_{\mathcal{K}, \mathbf{e}}}\left(c_{\mathcal{K}}^{n+1}-c_{\mathbf{e}}^{n+1}\right) u_{\mathcal{K}}+\frac{\sigma_{b}}{\varepsilon} \sum_{\mathcal{K} \in \mathfrak{M}} m_{\widetilde{\mathcal{K}}} d^{f_{b}}\left(c_{\mathcal{K}}^{n}, c_{\mathcal{K}}^{n+1}\right) u_{\mathcal{K}} \\
\frac{\varepsilon^{3}}{\Gamma_{b} \Gamma_{s}} \sum_{\mathbf{e} \in \partial \mathfrak{M}} m_{\widetilde{\mathbf{e}}} \frac{c_{\mathbf{e}}^{n+1}-c_{\mathbf{e}}^{n}}{\Delta t}= & -\varepsilon^{2} \sigma_{b} \sigma_{s} \llbracket c_{\partial \mathfrak{M}}^{n+1}, u_{\partial \mathfrak{M}} \rrbracket_{1, \partial \mathfrak{M}}-\sigma_{b} \sum_{\mathbf{e} \in \partial \mathfrak{M}} m_{\widetilde{\mathbf{e}}} d^{f_{s}}\left(c_{\mathbf{e}}^{n}, c_{\mathbf{e}}^{n+1}\right) u_{\mathbf{e}} \\
& -\varepsilon \sigma_{b} \sum_{\sigma \in \mathcal{E}_{e x t}} \frac{m_{\widetilde{\mathbf{e}}}}{d_{\mathcal{K}, \mathbf{e}}}\left(c_{\mathbf{e}}^{n+1}-c_{\mathcal{K}}^{n+1}\right) u_{\mathbf{e}}
\end{aligned}\right.
$$

The functions $d^{f_{b}}$ and $d^{f_{s}}$ represent the discretizations for nonlinear terms $f_{b}^{\prime}(c)$ and $f_{s}^{\prime}(c)$.

We can notice that in scheme $(\mathcal{S})$ the coupling between interior and boundary unknowns is performed by the two boxed terms: one in the interior mesh $\mathfrak{M}$ and the other on the boundary mesh $\partial \mathfrak{M}$.

We can also remark that scheme $(\mathcal{S})$ only uses geometric quantities related to the polygonal approximations of the control volumes. However, the convergence analysis is performed by using the exact geometric quantities related to the curved control volumes. In particular, we use projections of continuous functions on these curved control volumes which are useful to obtain a suitable approximation for the initial data.

In order to simplify the presentation and the analysis, we have written the scheme as a formulation which looks like a variational formulation. However, if for each control volume we choose the indicator function of this particular control volume as a test function in $(\mathcal{S})$, we recognize a usual finite-volume flux balance equation.

\subsection{Energy estimate}

Here we give the definition of the discrete energy and the corresponding estimate.

Definition 3.2 (Discrete free energy). The discrete free energy associated with the continuous free energy (1) is composed of a bulk energy $\mathcal{F}_{b, \mathcal{T}}$ and a surface energy $\mathcal{F}_{s, \partial \mathfrak{m}}$ such that for all $c_{\mathcal{T}} \in \mathbb{R}^{\mathcal{T}}$ :

$$
\mathcal{F}_{\mathcal{T}}\left(c_{\mathcal{T}}\right)=\mathcal{F}_{b, \mathcal{T}}\left(c_{\mathcal{T}}\right)+\mathcal{F}_{s, \partial \mathfrak{M}}\left(c_{\partial \mathfrak{M}}\right)
$$

where:

$$
\mathcal{F}_{b, \mathcal{T}}\left(c_{\mathcal{T}}\right)=\frac{\sigma_{b}}{\varepsilon} \sum_{\mathcal{K} \in \mathfrak{M}} m_{\widetilde{\mathcal{K}}} f_{b}\left(c_{\mathcal{K}}\right)+\frac{\varepsilon}{2} \sigma_{b}\left|c_{\mathcal{T}}\right|_{1, \mathcal{T}}^{2} \quad \text { and } \quad \mathcal{F}_{s, \partial \mathfrak{M}}\left(c_{\partial \mathfrak{M}}\right)=\sigma_{b} \sum_{\mathbf{e} \in \partial \mathfrak{M}} m_{\widetilde{\mathbf{e}}} f_{s}\left(c_{\mathbf{e}}\right)+\frac{\varepsilon^{2}}{2} \sigma_{b} \sigma_{s}\left|c_{\partial \mathfrak{M}}\right|_{1, \partial \mathfrak{M}}^{2} .
$$

By using Problem 3.1 with $v_{\mathfrak{M}}=\mu_{\mathfrak{M}}^{n+1}$ and $u_{\mathcal{T}}=c_{\mathcal{T}}^{n+1}-c_{\mathcal{T}}^{n}$ as test functions, we obtain the following energy estimate.

Proposition 3.3 (General energy estimate). Let $c_{\mathcal{T}}^{n} \in \mathbb{R}^{\mathcal{T}}$. We assume that there exists a solution $\left(c_{\mathcal{T}}^{n+1}, \mu_{\mathfrak{M}}^{n+1}\right)$ to Problem 3.1. Then, the following equality holds:

$$
\begin{aligned}
\mathcal{F}_{\mathcal{T}}\left(c_{\mathcal{T}}^{n+1}\right)- & \mathcal{F}_{\mathcal{T}}\left(c_{\mathcal{T}}^{n}\right)+\Delta t \Gamma_{b}\left|\mu_{\mathfrak{M}}^{n+1}\right|_{1, \mathcal{T}}^{2}+\frac{\varepsilon^{3}}{\Gamma_{b} \Gamma_{s}} \frac{1}{\Delta t}\left\|c_{\partial \mathfrak{M}}^{n+1}-c_{\partial \mathfrak{M}}^{n}\right\|_{0, \partial \mathfrak{M}}^{2} \\
+ & \frac{\varepsilon}{2} \sigma_{b}\left|c_{\mathcal{T}}^{n+1}-c_{\mathcal{T}}^{n}\right|_{1, \mathcal{T}}^{2}+\frac{\varepsilon^{2}}{2} \sigma_{b} \sigma_{s}\left|c_{\partial \mathfrak{M}}^{n+1}-c_{\partial \mathfrak{M}}^{n}\right|_{1, \partial \mathfrak{M}}^{2} \\
& =\frac{\sigma_{b}}{\varepsilon} \sum_{\mathcal{K} \in \mathfrak{M}} m_{\widetilde{\mathcal{K}}}\left(f_{b}\left(c_{\mathcal{K}}^{n+1}\right)-f_{b}\left(c_{\mathcal{K}}^{n}\right)-d^{f_{b}}\left(c_{\mathcal{K}}^{n}, c_{\mathcal{K}}^{n+1}\right)\left(c_{\mathcal{K}}^{n+1}-c_{\mathcal{K}}^{n}\right)\right) \\
& +\sigma_{b} \sum_{\mathbf{e} \in \partial \mathfrak{M}} m_{\widetilde{\mathbf{e}}}\left(f_{s}\left(c_{\mathbf{e}}^{n+1}\right)-f_{s}\left(c_{\mathbf{e}}^{n}\right)-d^{f_{s}}\left(c_{\mathbf{e}}^{n}, c_{\mathbf{e}}^{n+1}\right)\left(c_{\mathbf{e}}^{n+1}-c_{\mathbf{e}}^{n}\right)\right) .
\end{aligned}
$$




\subsection{Discretization for nonlinear terms}

In this section, we detail our two discretizations for the nonlinear terms used in scheme $(\mathcal{S})$ and we give the associated energy estimates.

\subsubsection{Fully implicit discretization}

For the fully implicit discretization in time, we choose $d^{f_{b}}$ and $d^{f_{s}}$ independent of $c_{\mathcal{T}}^{n}$ namely:

$$
d^{f_{b}}\left(c_{\mathcal{K}}^{n}, c_{\mathcal{K}}^{n+1}\right)=f_{b}^{\prime}\left(c_{\mathcal{K}}^{n+1}\right), \forall \mathcal{K} \in \mathfrak{M} \quad \text { and } \quad d^{f_{s}}\left(c_{\mathbf{e}}^{n}, c_{\mathbf{e}}^{n+1}\right)=f_{s}^{\prime}\left(c_{\mathbf{e}}^{n+1}\right), \forall \mathbf{e} \in \partial \mathfrak{M} .
$$

Then, by using the energy estimate $(2)$ and dissipativity assumptions $\left(\mathcal{H}_{\text {diss }}\right)$, we obtain the following discrete energy inequality:

Proposition 3.4 (Discrete energy inequality). Let $c_{\mathcal{T}}^{n} \in \mathbb{R}^{\mathcal{T}}$. We assume that there exists a solution $\left(c_{\mathcal{T}}^{n+1}, \mu_{\mathfrak{M}}^{n+1}\right)$ to Problem 3.1. Then, there exists $\Delta t_{0}>0$ such that for all $\Delta t \leq \Delta t_{0}$, we have:

$$
\begin{aligned}
\mathcal{F}_{\mathcal{T}}\left(c_{\mathcal{T}}^{n+1}\right)+ & \frac{\Delta t \Gamma_{b}}{2}\left|\mu_{\mathfrak{M}}^{n+1}\right|_{1, \mathcal{T}}^{2}+\frac{\varepsilon^{3}}{\Gamma_{b} \Gamma_{s}} \frac{1}{2 \Delta t}\left\|c_{\partial \mathfrak{M}}^{n+1}-c_{\partial \mathfrak{M}}^{n}\right\|_{0, \partial \mathfrak{M}}^{2} \\
& +\frac{\varepsilon}{4} \sigma_{b}\left|c_{\mathcal{T}}^{n+1}-c_{\mathcal{T}}^{n}\right|_{1, \mathcal{T}}^{2}+\frac{\varepsilon^{2}}{2} \sigma_{b} \sigma_{s}\left|c_{\partial \mathfrak{M}}^{n+1}-c_{\partial \mathfrak{M}}^{n}\right|_{1, \partial \mathfrak{M}}^{2} \leq \mathcal{F}_{\mathcal{T}}\left(c_{\mathcal{T}}^{n}\right) .
\end{aligned}
$$

We can notice that $\Delta t_{0}$ depends on the parameters of the equation, so with this discretization we have to choose a small enough $\Delta t$. This is why we introduce below another discretization.

\subsubsection{Semi-implicit discretization}

We would like to obtain an energy estimate without any condition on $\Delta t$. Thus, we choose a discretization for nonlinear terms such that the right hand side in (2) is equal to 0 :

$$
d^{f_{b}}(x, y)=\frac{f_{b}(y)-f_{b}(x)}{y-x} \quad \text { and } \quad d^{f_{s}}(x, y)=\frac{f_{s}(y)-f_{s}(x)}{y-x}, \quad \forall x, y .
$$

We can remark that the potentials used for numerical tests are polynomials. Thus, we can express $d^{f_{b}}$ (respectively $d^{f_{s}}$ ) as a polynomial in the variables $x, y$.

We thus obtain the following energy equality true for all $\Delta t>0$ :

Proposition 3.5 (Discrete energy equality). Let $c_{\mathcal{T}}^{n} \in \mathbb{R}^{\mathcal{T}}$. We assume that there exists a solution $\left(c_{\mathcal{T}}^{n+1}, \mu_{\mathfrak{M}}^{n+1}\right)$ to Problem 3.1, then we have:

$$
\begin{aligned}
\mathcal{F}_{\mathcal{T}}\left(c_{\mathcal{T}}^{n+1}\right) & +\Delta t \Gamma_{b}\left|\mu_{\mathfrak{M}}^{n+1}\right|_{1, \mathcal{T}}^{2}+\frac{\varepsilon^{3}}{\Gamma_{b} \Gamma_{s}} \frac{1}{\Delta t} \| c_{\partial \mathfrak{M}}^{n+1}-\left.c_{\partial \mathfrak{M}}^{n}\right|_{0, \partial \mathfrak{M}} ^{2} \\
& +\frac{\varepsilon}{2} \sigma_{b}\left|c_{\mathcal{T}}^{n+1}-c_{\mathcal{T}}^{n}\right|_{1, \mathcal{T}}^{2}+\frac{\varepsilon^{2}}{2} \sigma_{b} \sigma_{s}\left|c_{\partial \mathfrak{M}}^{n+1}-c_{\partial \mathfrak{M}}^{n}\right|_{1, \partial \mathfrak{M}}^{2}=\mathcal{F}_{\mathcal{T}}\left(c_{\mathcal{T}}^{n}\right) .
\end{aligned}
$$

We can notice that we use here two different discretizations for nonlinear potentials, but we can choose another discretization such as, for example, the convex-concave discretization (see [1] for more details).

\section{ExistenCE AND CONVERGENCE}

We give general assumptions on the discretization of nonlinear potential $d^{f_{b}}$ to demonstrate the existence and convergence theorems.

$d^{f_{b}}$ is of $\mathcal{C}^{1}$ class and there exist $C_{b} \geq 0$ and a real $p$ such that $2 \leq p<+\infty$,

$$
\left|d^{f_{b}}(a, b)\right| \leq C_{b}\left(1+|a|^{p-1}+|b|^{p-1}\right) \quad \text { and } \quad\left|D\left(d^{f_{b}}(a, .)\right)(b)\right| \leq C_{b}\left(1+|a|^{p-2}+|b|^{p-2}\right) . \quad\left(\mathcal{H}_{d^{f_{b}}}\right)
$$




\subsection{Existence}

The existence of a solution to discrete Problem 3.1 is based on the topological degree theory and the a priori energy estimates obtained above.

Theorem 4.1 (Existence of a discrete solution). Let $c_{\mathcal{T}}^{n} \in \mathbb{R}^{\mathcal{T}}$. Assuming that dissipativity assumptions $\left(\mathcal{H}_{\text {diss }}\right)$ and growth conditions $\left(\mathcal{H}_{d^{f_{b}}}\right)$ hold and that there exist constants $K_{b}^{c_{\mathcal{T}}^{n}}, K_{s}^{c_{\mathcal{T}}^{n}}$ (depending possibly on $c_{\mathcal{T}}^{n}$ ) such that, for all $u_{\mathcal{T}} \in \mathbb{R}^{\mathcal{T}}$,

$$
\begin{aligned}
& \sum_{\mathcal{K} \in \mathfrak{M}} m_{\widetilde{\mathcal{K}}}\left(f_{b}\left(u_{\mathcal{K}}\right)-f_{b}\left(c_{\mathcal{K}}^{n}\right)-d^{f_{b}}\left(c_{\mathcal{K}}^{n}, u_{\mathcal{K}}\right)\left(u_{\mathcal{K}}-c_{\mathcal{K}}^{n}\right)\right) \leq K_{b}^{c_{\mathcal{T}}^{n}}, \\
& \sum_{\mathbf{e} \in \partial \mathfrak{M}} m_{\widetilde{\mathbf{e}}}\left(f_{s}\left(u_{\mathbf{e}}\right)-f_{s}\left(c_{\mathbf{e}}^{n}\right)-d^{f_{s}}\left(c_{\mathbf{e}}^{n}, u_{\mathbf{e}}\right)\left(u_{\mathbf{e}}-c_{\mathbf{e}}^{n}\right)\right) \leq K_{s}^{c_{\mathcal{T}}^{n}} .
\end{aligned}
$$

Then, there exists at least one solution $\left(c_{\mathcal{T}}^{n+1}, \mu_{\mathfrak{M}}^{n+1}\right) \in \mathbb{R}^{\mathcal{T}} \times \mathbb{R}^{\mathfrak{M}}$ to Problem 3.1.

\subsection{Convergence}

In order to prove the convergence result we have to define a solution to Problem $(\mathcal{P})$ in a weak sense.

Definition 4.2 (Weak formulation). We say that a couple $(c, \mu) \in L^{\infty}\left(0, T ; H^{1}(\Omega)\right) \times L^{2}\left(0, T ; H^{1}(\Omega)\right)$ such that $\operatorname{Tr}(c) \in L^{\infty}\left(0, T ; H^{1}(\Gamma)\right)$ is solution to continuous Problem $(\mathcal{P})$ in the weak sense if for all $\psi \in \mathcal{C}_{c}^{\infty}([0, T[\times \bar{\Omega})$, the following identities hold:

$$
\begin{aligned}
& \int_{0}^{T} \int_{\Omega}\left(-\partial_{t} \psi c+\Gamma_{b} \nabla \mu \cdot \nabla \psi\right)=\int_{\Omega} c^{0} \psi(0, .) \\
& \int_{0}^{T} \int_{\Omega}\left(-\mu \psi+\varepsilon \sigma_{b} \nabla c \cdot \nabla \psi+\frac{\sigma_{b}}{\varepsilon} f_{b}^{\prime}(c) \psi\right)+\int_{0}^{T} \int_{\Gamma}\left(-\frac{\varepsilon^{3}}{\Gamma_{b} \Gamma_{s}} \partial_{t} \psi c_{\Gamma}+\sigma_{s} \sigma_{b} \varepsilon^{2} \nabla_{\|} c_{\Gamma} \cdot \nabla_{\|} \psi+\sigma_{b} f_{s}^{\prime}\left(c_{\Gamma}\right) \psi\right) \\
& \quad=\frac{\varepsilon^{3}}{\Gamma_{b} \Gamma_{s}} \int_{\Gamma} \operatorname{Tr}\left(c^{0}\right) \psi(0, .) .
\end{aligned}
$$

Theorem 4.3 (Bounds of the solutions). Assuming that assumptions $\left(\mathcal{H}_{\text {diss }}\right),\left(\mathcal{H}_{f_{b}}\right),\left(\mathcal{H}_{d^{f_{b}}}\right),(3)$ hold and that there exists a constant $C>0$ such that, for all $n \in \mathbb{N}$,

$$
\begin{aligned}
\mathcal{F}_{\mathcal{T}}\left(c_{\mathcal{T}}^{n+1}\right) & +C\left(\Delta t \Gamma_{b}\left|\mu_{\mathfrak{M}}^{n+1}\right|_{1, \mathcal{T}}^{2}+\frac{\varepsilon^{3}}{\Gamma_{b} \Gamma_{s}} \frac{1}{\Delta t} \| c_{\partial \mathfrak{M}}^{n+1}-\left.c_{\partial \mathfrak{M}}^{n}\right|_{0, \partial \mathfrak{M}} ^{2}+\frac{\varepsilon}{2} \sigma_{b}\left|c_{\mathcal{T}}^{n+1}-c_{\mathcal{T}}^{n}\right|_{1, \mathcal{T}}^{2}+\frac{\varepsilon^{2}}{2} \sigma_{b} \sigma_{s}\left|c_{\partial \mathfrak{M}}^{n+1}-c_{\partial \mathfrak{M}}^{n}\right|_{1, \partial \mathfrak{M}}^{2}\right) \\
\leq & \mathcal{F}_{\mathcal{T}}\left(c_{\mathcal{T}}^{n}\right),
\end{aligned}
$$

then, there exists $M>0$ independent of $\mathcal{T}$ and $\Delta t$ such that:

$$
\begin{gathered}
\sup _{n \leq N}\left\|c_{\mathcal{T}}^{n}\right\|_{1, \mathcal{T}} \leq M, \quad \sup _{n \leq N}\left\|c_{\partial \mathfrak{M}}^{n}\right\|_{1, \partial \mathfrak{M}} \leq M, \quad \sum_{n=0}^{N-1} \Delta t\left\|\mu_{\mathfrak{M}}^{n+1}\right\|_{1, \mathcal{T}}^{2} \leq M, \\
\Delta t \sum_{n=0}^{N-1} \Delta t\left\|\frac{c_{\mathcal{T}}^{n+1}-c_{\mathcal{T}}^{n}}{\Delta t}\right\|_{1, \mathcal{T}}^{2} \leq M \quad \text { and } \quad \Delta t \sum_{n=0}^{N-1} \Delta t\left\|\frac{c_{\partial \mathfrak{M}}^{n+1}-c_{\partial \mathfrak{M}}^{n}}{\Delta t}\right\|_{1, \partial \mathfrak{M}}^{2} \leq M .
\end{gathered}
$$

These bounds are one of the key elements to prove the convergence result below by using the discrete $H^{1}$ compactness and the Kolmogorov theorem. We also note that we have nonlinearities in the domain $\Omega$ and on the boundary $\Gamma$. Thus $L^{2}((0, T) \times \Omega)$ compactness is not sufficient and we have to prove uniform estimates of time and space translates on $\Omega$ and $\Gamma$. 
Theorem 4.4 (Estimation of time and space translates). Let $\left(c_{\mathcal{T}}^{\Delta t}, \mu_{\mathfrak{M}}^{\Delta t}\right)$ be a solution to Problem 3.1, then there exists $C>0$ (not depending on $\operatorname{size}(\mathcal{T})$ and $\Delta t$ ) such that:

$$
\begin{aligned}
& \left\|\widetilde{c_{\mathfrak{M}} t}(.+\tau, .)-\widetilde{c_{\mathfrak{M}}^{\triangle t}}(., .)\right\|_{L^{2}\left(\mathbb{R} \times \mathbb{R}^{2}\right)}^{2} \leq C \tau, \quad\left\|\widetilde{c_{\mathfrak{M}}^{\Delta t}}(., .+\eta)-\widetilde{c_{\mathfrak{M}}^{\Delta t}}(., .)\right\|_{L^{2}\left(\mathbb{R} \times \mathbb{R}^{2}\right)}^{2} \leq C|\eta|, \\
& \left\|\widetilde{c_{\partial \mathfrak{M}}^{\Delta t}}(.+\tau, .)-\widetilde{c_{\partial \mathfrak{M}}^{\Delta t}}(., .)\right\|_{L^{2}(\mathbb{R} \times \Gamma)}^{2} \leq C \tau, \quad\left\|\widetilde{c_{\partial \mathfrak{M}}^{\triangle t}}\left(., \tau_{\eta}(.)\right)-\widetilde{c_{\partial \mathfrak{M}}^{\Delta t}}(., .)\right\|_{L^{2}(\mathbb{R} \times \Gamma)}^{2} \leq C|\eta|,
\end{aligned}
$$

where we note $\widetilde{c_{\mathfrak{M}}^{\Delta t}}$ (respectively $\widetilde{c_{\partial \mathfrak{M}}^{\Delta t}}$ ) the extension by 0 of $c_{\mathfrak{M}}^{\Delta t}$ (respectively $c_{\partial \mathfrak{M}}^{\Delta t}$ ) on $\mathbb{R} \times \mathbb{R}^{2}$ (respectively $\left.\mathbb{R} \times \Gamma\right)$ and $\tau_{\eta}$ represents the shifting of length $\eta$ along the boundary $\Gamma$ (an orientation being given on $\Gamma$ ).

Theorem 4.4 is proved by using the bounds of the solutions given by Theorem 4.3 and scheme $(\mathcal{S})$. This Theorem is essential to apply the Kolmogorov theorem and to prove the following convergence result.

Theorem 4.5 (Convergence theorem). Assuming that conditions $\left(\mathcal{H}_{\text {diss }}\right),\left(\mathcal{H}_{f_{b}}\right),\left(\mathcal{H}_{d^{f_{b}}}\right),(3)$ and $(6)$ hold, let us consider Problem $(\mathcal{P})$ with an initial condition $c_{0} \in H^{1}(\Omega)$ such that $\operatorname{Tr}\left(c_{0}\right) \in H^{1}(\Gamma)$. Then, there exists a weak solution $(c, \mu)$ on $\left[0, T\left[\right.\right.$ (in the sense of Definition 4.2). Furthermore, let $\left(c^{(m)}, c_{\Gamma}^{(m)}\right)_{m \in \mathbb{N}}$ and $\left(\mu^{(m)}\right)_{m \in \mathbb{N}}$ be a sequence of solutions to Problem 3.1 with a sequence of discretizations such that the space and time steps, $h_{\mathcal{T}}^{(m)}$ and $\Delta t^{(m)}$ respectively, tend to 0 . Then, up to a subsequence, the following convergence properties hold, for all $q \geq 1$, when $h_{\mathcal{T}}^{(m)}, \Delta t^{(m)} \rightarrow 0$ :

$$
\begin{aligned}
c^{(m)} & \rightarrow c \text { in } L^{2}\left(0, T ; L^{q}(\Omega)\right) \text { strongly, } \\
c_{\Gamma}^{(m)} & \rightarrow \operatorname{Tr}(c) \text { in } L^{2}\left(0, T ; L^{q}(\Gamma)\right) \text { strongly, } \\
\text { and } \mu^{(m)} & \rightarrow \mu \text { in } L^{2}\left(0, T ; L^{q}(\Omega)\right) \text { weakly. }
\end{aligned}
$$

Remark 4.6. We chose the initial concentration in the scheme equal to the mean-value projection:

$$
c_{\mathcal{T}}^{0}=\left(\left(\frac{1}{m_{\mathcal{K}}} \int_{\mathcal{K}} c_{0}\right)_{\mathcal{K} \in \mathfrak{M}},\left(\frac{1}{m_{\mathbf{e}}} \int_{\mathbf{e}} c_{0}\right)_{\mathbf{e} \in \partial \mathfrak{M}}\right) .
$$

\section{Numerical Simulations}

In this section, we present numerical experiments for three different nonlinear surface free energy densities. We choose here the semi-implicit discretization in time for nonlinear terms in order to allow for a not too small time step $\Delta t$. For each simulation we consider the usual double-well bulk potential $f_{b}(c)=c^{2}(1-c)^{2}$.

We are interested here by two domains with a Delaunay triangular mesh:

- a $(0,8) \times(0,4)$ rectangle with periodic boundary conditions in the lateral direction and dynamic boundary conditions in the vertical direction;

- a smooth curved domain whose diameter is equal to 2 and with dynamic boundary conditions everywhere on $\Gamma$.

In these cases, $x_{\mathcal{K}}$ is the circumcenter of the control volume $\mathcal{K}$ and $y_{\mathbf{e}}$ is the middle of the chord $\widetilde{\mathbf{e}}$.

For each domain, we choose a random initial $c^{0} \in \mathbb{R}^{\mathcal{T}}$ between 0.4 and 0.6 and we keep this initial data for each simulation with the same domain. We can then observe the influence of the boundary conditions on the phase separation dynamics.

\subsection{Influence of the surface diffusion term}

First, we choose $f_{s}=f_{b}$ for the surface potential and to compare the results in $[5,6]$, we begin with the rectangular domain (Fig. 3) and the following parameters: $\varepsilon=0.3, \Gamma_{b}=\sigma_{b}=0.1$ for the bulk, $\Gamma_{s}=10$ for the 


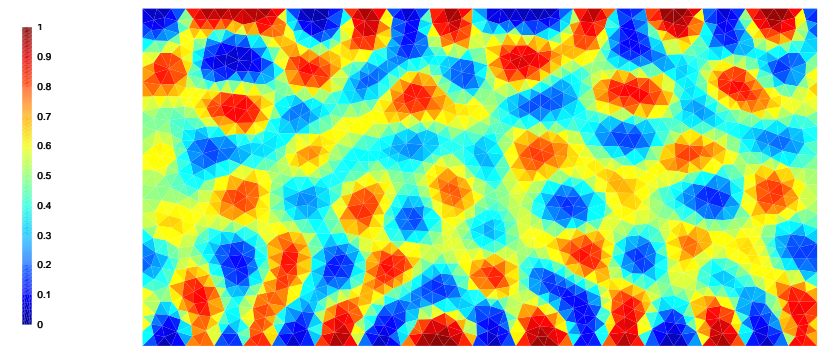

(A) $\sigma_{s}=0$

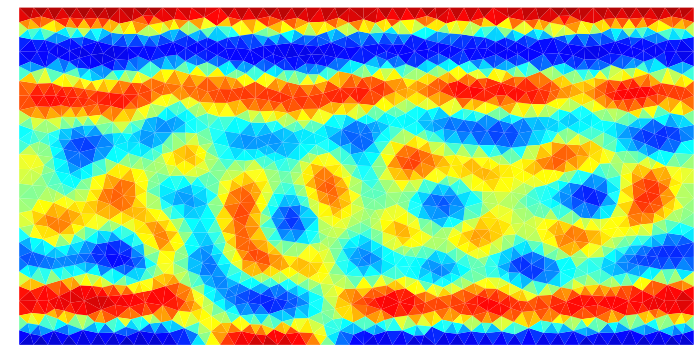

(в) $\sigma_{s}=5$

FiguRE 3. Spinodal decomposition for rectangular domain

surface and $T=0.75, \mathrm{~d} t=0.05$ for the time. We observe the influence of surface diffusion by computing the solution with two different values for the surface coefficient $\sigma_{s}$.

In both case, we have lateral anisotropic structures but their length scale is different. Indeed, when we have $\sigma_{s}=0$ (Fig. 3a) the structure length scales are shorter than when we have $\sigma_{s}=5$ (Fig. 3b). These results are very close to those observed in [5].

Now, we test the scheme with our curved domain (Fig. 4) with the following parameters: $\varepsilon=\Gamma_{b}=\sigma_{b}=0.1$ for the bulk, $\Gamma_{s}=10$ for the surface and $T=0.025, \mathrm{~d} t=0.005$ for the time.

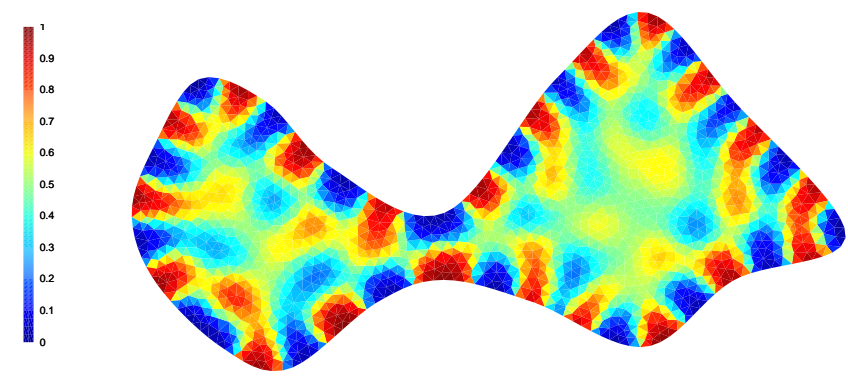

(A) $\sigma_{s}=0$

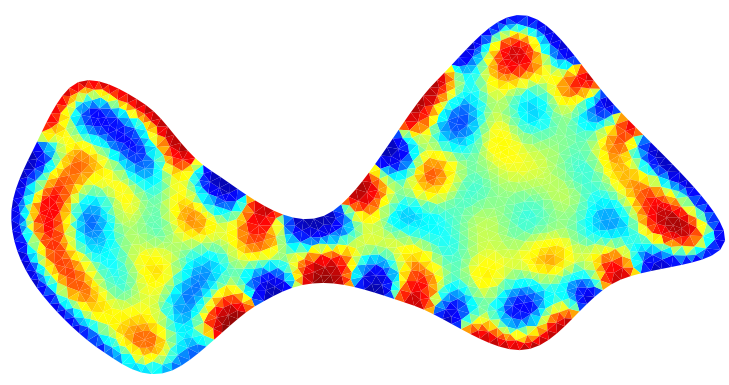

(в) $\sigma_{s}=5$

Figure 4. Spinodal decomposition for curved domain

We observe the same behavior as for the rectangular domain (Fig. 3): for $\sigma_{s}=0$ (Fig. 4a), we have small typical structures on the boundary while for $\sigma_{s}=5$ (Fig. 4a) the structures are large excepted where the domain is too narrow.

\subsection{Preferential attraction by the wall}

For the following computation (Fig 5), we want to observe the influence of the surface potential by taking $f_{s}(c)=g_{s} c^{2}-\left(h_{s}+g_{s}\right) c$ where $h_{s} \neq 0$ describes the possible preferential attraction of one of the two components by the wall. Thus, we choose fixed parameters: $\varepsilon=0.2, \Gamma_{b}=\sigma_{b}=0.1$ for the bulk, $\Gamma_{s}=10, \sigma_{s}=0, g_{s}=10$ for the surface and $T=0.37, \mathrm{~d} t=0.001$ for the time and we modify the coefficient $h_{s}$.

First, we notice than the parallel structures observed when $h_{s}=0$ (Fig. 5a) are similar to those observed in $[2,7]$. Then, we confirm the preferential attraction of the phase $c=1$ by the boundary when $h_{s}>0$ (Fig. 5b) and we notice that this attraction changes all the behavior in the domain $\Omega$. 


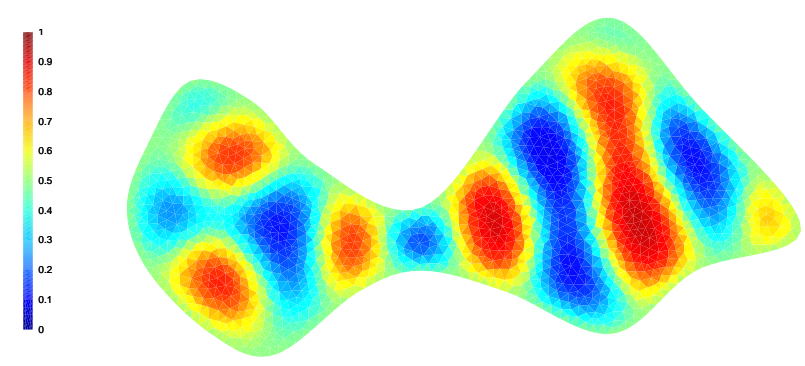

(A) $h_{s}=0$

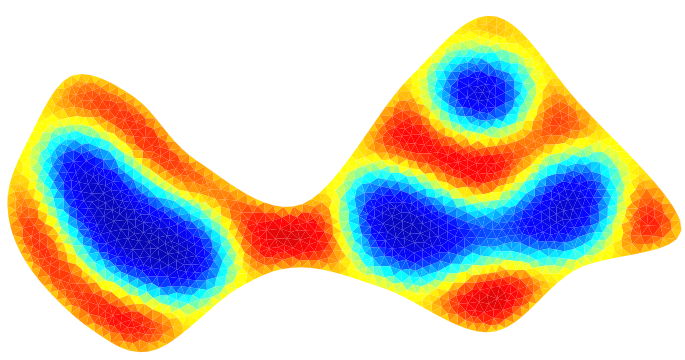

(в) $h_{s}=3$

FiguRE 5. Influence of $h_{s}$

\section{CONCLUSION}

We propose here a finite-volume scheme to deal with the 2D Cahn-Hilliard equation with dynamic boundary conditions. With this method the coupling between the equation in the domain and the equation on the boundary is easy to implement even with a curved geometry for the mesh. Furthermore, we give a convergence result which additionally enables to obtain the existence of weak solutions for the continuous problem.

We can specify that we have error estimates for the Cahn-Hilliard equation with Neumann boundary conditions. Moreover, in [8] we performed numerical simulations for error estimates which gave the expected first-order convergence.

Finally, a possible future work will be the coupling of Cahn-Hilliard system $(\mathcal{P})$ with the Navier-Stokes equation, such as, for example, in [3].

\section{REFERENCES}

[1] F. Boyer and S. Minjeaud. Numerical schemes for a three component Cahn-Hilliard model. ESAIM Math. Model. Numer. Anal., 45(4):697-738, 2011.

[2] L. Cherfils, M. Petcu, and M. Pierre. A numerical analysis of the Cahn-Hilliard equation with dynamic boundary conditions. Discrete Contin. Dyn. Syst., 27(4):1511-1533, 2010.

[3] S. Dong. On imposing dynamic contact-angle boundary conditions for wall-bounded liquid-gas flows. Comput. Methods Appl. Mech. Engrg., 247/248:179-200, 2012.

[4] R. Eymard, T. Gallouët, and R. Herbin. Finite volume methods. Handb. Numer. Anal., VII. North-Holland, Amsterdam, 2000.

[5] H.P. Fischer, P. Maass, and W. Dieterich. Novel surface modes in spinodal decomposition. Phys. Rev. Lett., 79:893-896, Aug 1997.

[6] H.P. Fischer, P. Maass, and W. Dieterich. Diverging time and length scales of spinodal decomposition modes in thin films. EPL (Europhysics Letters), 42(1):49-54, 1998.

[7] R. Kenzler, F. Eurich, P. Maass, B. Rinn, J. Schropp, E. Bohl, and W. Dieterich. Phase separation in confined geometries: Solving the Cahn-Hilliard equation with generic boundary conditions. j-COMP-PHYS-COMM, 133:139-157, Jan 2001.

[8] F. Nabet. Finite volume analysis for the Cahn-Hilliard equation with dynamic boundary conditions. In Finite volumes for complex applications. VII. Methods and Theoretical aspects, Springer Proc. Math. to appear, 2014. http://hal. archives-ouvertes.fr/hal-00974585.

[9] J. Prüss, R. Racke, and S. Zheng. Maximal regularity and asymptotic behavior of solutions for the Cahn-Hilliard equation with dynamic boundary conditions. Ann. Mat. Pura Appl. (4), 185(4):627-648, 2006.

[10] R. Racke and S. Zheng. The Cahn-Hilliard equation with dynamic boundary conditions. Adv. Differential Equations, 8(1):83$110,2003$.

[11] H. Wu and S. Zheng. Convergence to equilibrium for the Cahn-Hilliard equation with dynamic boundary conditions. J. Differential Equations, 204(2):511-531, 2004. 